\title{
High Performance Liquid Chromatographic Determination of Acidic Catecholamine Metabolites in Human Urine Using Postcolumn Fluorescence Derivatization with $\boldsymbol{d l}$-1,2-Diphenylethylenediamine
}

\author{
Hee-Kyoung JeON, Hitoshi NohTA and Yosuke OHKURA \\ Faculty of Pharmaceutical Sciences, Kyushu University 62, Maidashi, Fukuoka 812, Japan
}

\begin{abstract}
$d l$-1,2-Diphenylethylenediamine was found to react sensitively in an alkaline solution with 3,4-dihydroxymandelic acid as well as other catechol compounds and 4-hydroxy-3-methoxyphenyl compounds to yield fluorescent products. By using this reaction, a postcolumn fluorescence derivatization high performance liquid chromatography of the acidic metabolites of catecholamines (3,4-dihydroxymandelic acid, 3,4-dihydroxyphenylacetic acid, vanillylmandelic acid and homovanillic acid) in human urine was established. These compounds were separated on an anion-exchanger column (TSK-gel DEAE-2SW) and subjected to derivatization involving coulometric oxidation followed by a fluorescence reaction with the named reagent. The detection limits $(S / N=3)$ for the acidic metabolites in urine were $15-30 \mathrm{fmol}$ in a $100-\mu l$ injection volume.
\end{abstract}

Keywords dl-1,2-Diphenylethylenediamine, acidic metabolite, catecholamine, 3,4-dihydroxymandelic acid, high performance liquid chromatography, fluorescence derivatization, electrochemical oxidation, human urine

Catecholamines (norepinephrine, epinephrine and dopamine) are metabolized and excreted mainly as phenylacetic acid derivatives ${ }^{1}$ [3,4-dihydroxymandelic acid (DOMA), 3,4-dihydroxyphenylacetic acid (DOPAC), vanillylmandelic acid (VMA) and homovanillic acid (HVA)] and their conjugates. Urinary HVA and VMA, the major acidic metabolites, have been used for the diagnosis of nuroblastoma ${ }^{2-4}$, pheochromocytoma ${ }^{2,4}$ and melanoma. 5,6 The minor acidic metabolites of catecholamines, DOPAC ${ }^{1}$ and DOMA ${ }^{1,2}$, are also excreted in urine. Thus, the total excretion of the acidic metabolites reflects well the overall turnover of catecholamines and is of clinical value in screening diseases affecting catecholamine production.

Many assay methods regarding acidic metabolites have been reported: coulometry ${ }^{7,8}$, fluorometry ${ }^{9-11}$, gas chromatography coupled with mass spectrometry (GCMS) ${ }^{12,13}$ and high performance liquid chromatography (HPLC) with ultraviolet ${ }^{14,15}$, fluorescence ${ }^{16}$ or electrochemical ${ }^{17-19}$ detection. The coulometric and fluorometric methods are not very satisfactory regarding both sensitivity and selectivity when applied to biological samples. Though GC-MS methods can give the most reliable results, they require sophisticated equipment. In recent years, HPLC has been accepted as a facile tool for the determination of the urinary acidic metabolites of catecholamines, where biological samples should be cleaned by chromatography on an anionexchanger ${ }^{20}$ or reversed-phase ${ }^{21}$ short column, or by a solvent-extraction procedure. ${ }^{18,22}$ These clean-up procedures are time consuming and often result in low recoveries of the metabolites $(48-80 \%)$.
We have reported an ion-pair reversed-phase HPLC ${ }^{23}$ for the determination of catechol compounds and 4hydroxy-3-methoxyphenyl compounds by postcolumn derivatization involving coulometric oxidation of the compounds to the corresponding $o$-quinones followed by fluorescence reaction using meso-1,2-diphenylethylenediamine (meso-DPE). ${ }^{24}$ Though the meso-DPE reaction was highly sensitive to catecholamines, it was two orders of magnitude less sensitive to DOMA. In this study we first found that $d l-1,2$-diphenylethylenediamine ( $d l$-DPE) can afford more intense fluorescence to DOMA, as well as DOPAC and catecholamines by screening of 1,2-diarylethylenediamines (DAEs). By using this result, a selective and highly sensitive method was established for the determination of the acidic metabolites of catecholamines by postcolumn derivatization HPLC. The acidic metabolites were separated by anion-exchange chromatography and oxidized to the respective $o$-quinones by a coulometric technique; the resulting $o$-quinones were fluorescence-derivatized with $d l$-DPE. The established method was then applied to human urine sample.

\section{Experimental}

\section{Reagents, solutions and apparatus}

DOMA, VMA and HVA were purchased from Nacalai Tesque (Kyoto, Japan). DOPAC was obtained from Aldrich (Milwaukee, USA). Their standard solutions were prepared in water and stored at $-20^{\circ} \mathrm{C}$. The standard solutions of other catechol and 4hydroxy-3-methoxyphenyl compounds were prepared 
Table 1 Excitation and emission maxima $\left(\lambda_{\mathrm{ex}}, \lambda_{\mathrm{em}}\right)$ of the fluorescence from DOMA and DOPAC with DAEs, and their relative fluorescence intensities $(\mathrm{RFI})^{\mathrm{a}}$

\begin{tabular}{|c|c|c|c|c|c|c|}
\hline \multirow{2}{*}{ DAE } & \multicolumn{3}{|c|}{ DOMA } & \multicolumn{3}{|c|}{ DOPAC } \\
\hline & $\lambda_{\text {ex }} / \mathrm{nm}$ & $\lambda_{\mathrm{em}} / \mathrm{nm}$ & RFI $^{\text {b }}$ & $\lambda_{\text {ex }} / \mathrm{nm}$ & $\lambda_{\mathrm{em}} / \mathrm{nm}$ & $\mathbf{R F I}^{\mathrm{b}}$ \\
\hline \multicolumn{7}{|l|}{$d l$ form } \\
\hline$d l$-DPE & 420 & 515 & 100 & 400 & 520 & 200 \\
\hline 1,2-Bis(4-methyl)ethylenediamine & 390 & 490 & 34 & 397 & 505 & 75 \\
\hline 1,2-Bis(4-methoxyphenyl)ethylenediamine & 390 & 485 & 25 & 390 & 485 & 27 \\
\hline \multicolumn{7}{|l|}{ meso form } \\
\hline meso-DPE & 400 & 500 & 27 & 365 & 470 & 373 \\
\hline 1,2-Bis(4-methoxyphenyl)ethylenediamine & 395 & 495 & 26 & 400 & 505 & 324 \\
\hline 1,2-Bis(4-chlorophenyl)ethylenediamine & 390 & 480 & 26 & 380 & 450 & 81 \\
\hline 1,2-Bis(3,4-dimethoxyphenyl)ethylenediamine & 370 & 465 & 25 & 365 & 440 & 30 \\
\hline 1,2-Bis(4-fluorophenyl)ethylenediamine & 400 & 505 & 24 & 405 & 515 & 204 \\
\hline 1,2-Bis(3,4-dimethylphenyl)ethylenediamine & 390 & 475 & 13 & 390 & 465 & 14 \\
\hline
\end{tabular}

a. Portions $(0.1 \mathrm{ml})$ of $10 \mathrm{nmol} \mathrm{m}^{-1}$ DOMA and DOPAC solutions were treated according to the procedure. b. The fluorescence intensity of the $d l$-DPE derivative of DOMA was taken as 100 .

and stored in the same way. The following 26 DAEs were synthesized as described previously: ${ }^{25}$ meso-DPE, 1,2-bis(2-hydroxyphenyl)ethylenediamine, 1,2-bis(4-methoxyphenyl)ethylenediamine, 1,2-bis(2- and 3-methoxyphenyl)ethylenediamines, 1,2-bis(2- and 4-ethoxyphenyl)ethylenediamines, 1,2-bis(2-, 3- and 4-methylphenyl)ethylenediamines, 1,2-bis(3,4-dimethoxyphenyl)ethylenediamine, 1,2-bis(3,4-methylenedioxyphenyl)ethylenediamine, 1,2-bis(4-cyanophenyl)ethylenediamines, 1,2-bis(2-, 3- and 4-chlorophenyl)ethylenediamines, 1,2-bis(2,6and 3,4-dichlorophenyl)ethylenediamines, 1,2-bis(4-fluorophenyl)ethylenediamine, 1,2-bis(biphenylyl)ethylenediamine, 1,2-bis(1- and 2-naphthyl)ethylenediamines, all in the meso form; and dl-DPE, 1,2-bis(4-methoxyphenyl)ethylenediamine and 1,2-bis(4-methylphenyl)ethylenediamine, all in the $d l$ form. Each DAE solution $(0.1 \mathrm{M})$ was prepared in ethanol. All other chemicals were of reagent grade. Deionized and distilled water was used.

Human urine (24-h) was collected in the presence of $10 \mathrm{ml}$ of $6 \mathrm{M}$ hydrochloric acid and stored at $-20^{\circ} \mathrm{C}$ until the assay.

Uncorrected fluorescence spectra were measured with a Hitachi MPF-4 spectrofluorometer using semimicro quartz cells (10-mm length parallel to the excitation beam, 3-mm width parallel to emission beam, $1 \mathrm{ml}$ ): spectral bandwidths of $10 \mathrm{~nm}$ were used in both the excitation and emission monochromators.

\section{Procedure for fluorescence reaction of catechol compounds} with DAEs (screening of DAEs)

To $0.1 \mathrm{ml}$ of aqueous test solution, $0.8 \mathrm{ml}$ of $30 \%$ acetonitrile, $0.1 \mathrm{ml}$ each of $0.1 \mathrm{M}$ DAE solution and $5 \mathrm{mM}$ sodium hydroxide were successively added in a screw-capped $3.5-\mathrm{ml}$ reaction vial. The vial was tightly closed and heated at $100^{\circ} \mathrm{C}$ for $60 \mathrm{~min}$, and then cooled in water. To prepare the blank, the same procedure was followed, except that the $0.1 \mathrm{ml}$ of the test solution was replaced with $0.1 \mathrm{ml}$ of water. The fluorescence intensities of the test and blank solutions were measur-
Table 2 Fluorescence excitation and emission maxima $\left(\lambda_{\mathrm{ex}}, \lambda_{\mathrm{em}}\right)$ and relative intensities (RFI) of the reaction mixtures of catechol and 4-hydroxy-3-methoxyphenyl compounds (other than DOMA and DOPAC) with dl-DPEa

\begin{tabular}{lrrr}
\hline \multicolumn{1}{c}{ Compound } & $\lambda_{\text {ex }} / \mathrm{nm}$ & $\lambda_{\text {em }} / \mathrm{nm}$ & RFI $^{\mathrm{b}}$ \\
\hline Catechol compounds & & & \\
Norepinephrine & 400 & 510 & 55 \\
Epinephrine & 400 & 470 & 54 \\
Dopamine & 370 & 475 & 25 \\
L-DOPA & 400 & 460 & 25 \\
3,4-Dihydroxyphenylglycol & 400 & 520 & 172 \\
3,4-Dihydroxybenzylamine & 400 & 525 & 209 \\
3,4-Dihydroxycinnamic acid & 380 & 505 & 106 \\
Isoproterenol & 400 & 510 & 77 \\
Carbidopa & 400 & 505 & 65 \\
L-3,4-Dihydroxyphenylserine & 405 & 510 & 16 \\
$\alpha$-Methyldopa & 400 & 510 & 22 \\
Protocatechuic acid & 400 & 525 & 220 \\
Protocatechualdehyde & 420 & 530 & 103 \\
3-Methylcatechol & 400 & 535 & 192 \\
Progallol & 410 & 500 & 340 \\
Gallic acid & 390 & 450 & 27 \\
Tiron & 400 & 500 & 78 \\
Esculetin & 400 & 470 & 945 \\
Quinic acid & 400 & 490 & 102 \\
Morin & 410 & 500 & 76
\end{tabular}

4-Hydroxy-3-methoxyphenyl compounds

\begin{tabular}{lllr} 
Normetanephrine & 415 & 525 & 50 \\
Metanephrine & 400 & 495 & 20 \\
3-Methoxytyramine & 420 & 520 & 3 \\
VMA & 400 & 525 & 12 \\
$\begin{array}{l}\text { HVA } \\
\text { 4-Hydroxy-3-methoxyphenyl- }\end{array}$ & 415 & 525 & 59 \\
$\begin{array}{l}\text { glycol } \\
\text { 4-Hydroxy-3-methoxyphenyl- } \\
\quad \text { ethanol }\end{array}$ & 400 & 525 & 122 \\
$\begin{array}{l}\text { 4-Hydroxy-3-methoxycin- } \\
\text { namic acid }\end{array}$ & 410 & 520 & 11 \\
\hline
\end{tabular}

a. Portions $(0.1 \mathrm{ml})$ of $10 \mathrm{nmol} \mathrm{m}^{-1}$ solutions of the compounds were treated according to the procedure. $b$. The intensity from DOMA with $d l$-DPE was taken as 100. 


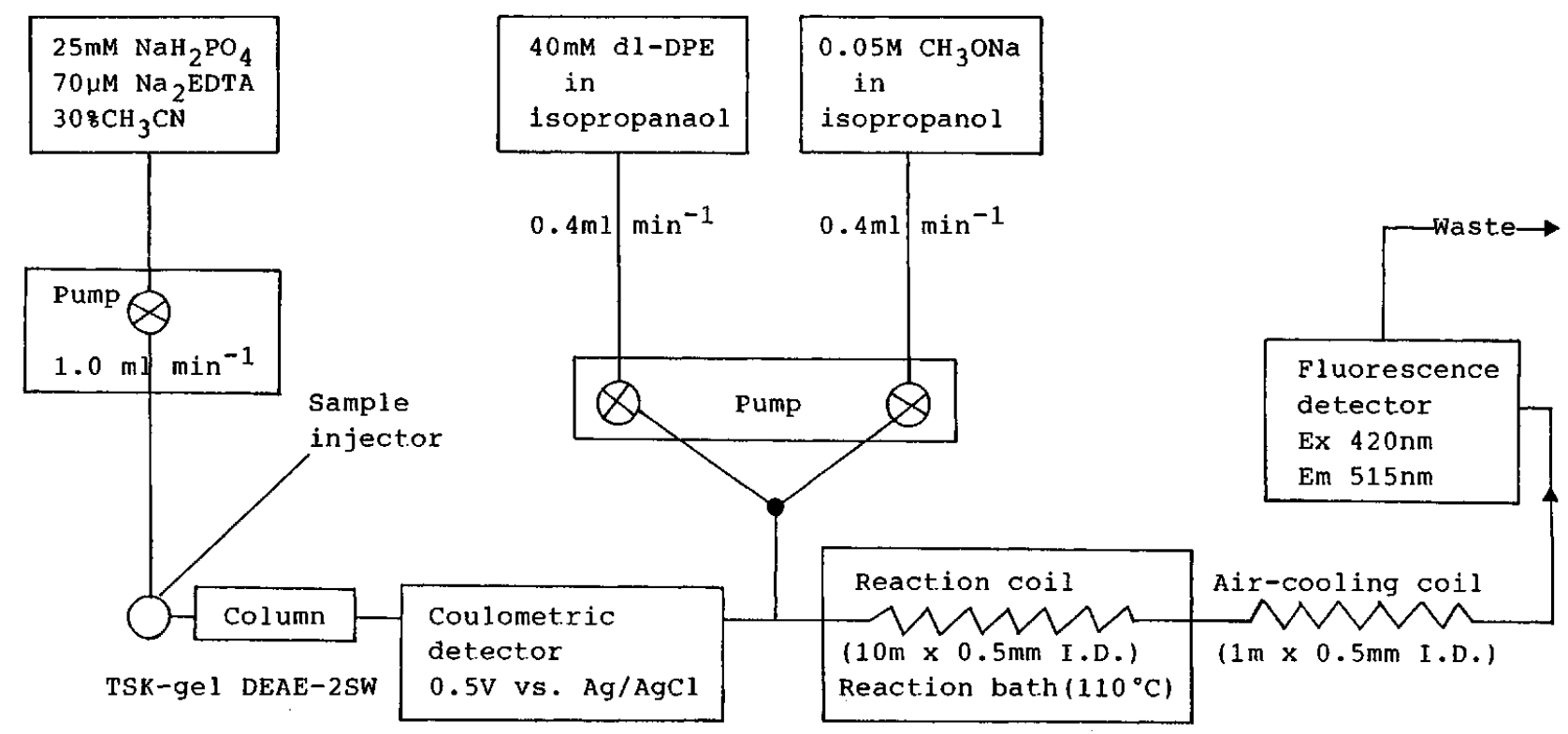

Fig. 1 Schematic diagram of the postcolumn fluorescence derivatization HPLC system.

ed at the respective excitation and emission maxima (see Tables 1 and 2).

\section{HPLC system and its operation conditions}

Figure 1 shows a schematic diagram of the HPLC system. An anion-exchanger column, TSK-gel DEAE2SW $(250 \times 4.6 \mathrm{~mm}$ i.d., particle size $5 \mu \mathrm{m}$; Tosoh, Tokyo, Japan) was used. The column temperature was ambient. The mobile phase was a mixture of $25 \mathrm{mM}$ sodium dihydrogenphosphate (pH 3.0) containing 70 $\mu \mathrm{M}$ disodium EDTA and acetonitrile $(7: 3, v / v)$. The flow rate was $1.0 \mathrm{ml} \mathrm{min}^{-1}$. The eluate from the column was introduced to a Coulochem $5100 \mathrm{~A}$ detector (Environmental Science Association, Inc., Bedford, USA) fitted with a model 5011 cell, which was set at the potential of $0.5 \mathrm{~V}$ versus $\mathrm{Ag} / \mathrm{AgCl}$ electrode.

The effluent from the coulometric detector was then mixed with a stream of a mixture of two reagent solutions: $40 \mathrm{mM} d l$-DPE in 2-propanol and $50 \mathrm{mM}$ sodium methylate in 2-propanol. The reagent solutions were both delivered at a flow rate of $0.4 \mathrm{ml} \mathrm{min}^{-1}$ by an SSP DM2M-1026 pump (Sanuki Kogyo, Tokyo, Japan). The fluorescence derivatization reaction proceeded in a reaction coil $(10 \mathrm{~m} \times 0.5 \mathrm{~mm}$ i.d., stainlesssteel tube) placed in a Tosoh RE-8010 reaction bath $\left(110^{\circ} \mathrm{C}\right)$. The effluent from the reaction coil was passed through an air-cooling coil $(1 \mathrm{~m} \times 0.5 \mathrm{~mm}$ i.d., stainlesssteel tube). The resulting fluorescence was monitored at $515 \mathrm{~nm}$ with an excitation wavelength of $420 \mathrm{~nm}$ by using a Hitachi 650-10 LC spectrofluorometer equipped with an 18- $\mu$ l flow cell. The spectral bandwidths for both the excitation and emission wavelengths were $10 \mathrm{~nm}$. The peak heights were used for quantification.

\section{Urine sample preparation for HPLC}

An aliquot $(100 \mu \mathrm{l})$ of urine $(24-\mathrm{h})$ was diluted 5-fold with water, and then ultrafiltered through a membrane filter (ULTRAFREE; molecular weight of exclusion limit, 10000; Nihon Millipore, Tokyo, Japan). A 100- $\mu 1$ aliquot of the filtrate was injected into the HPLC system.

\section{Results and Discussion}

Fluorescence reaction of catechol and 4-hydroxy-3-methoxyphenyl compounds with dl-DPE

Selection of $d l$-DPE and its fluorescence reaction. The screening of DAEs was carried out using DOMA and DOPAC. Of the 26 DAEs examined, 9 DAEs gave fairly intense fluorescence to DOMA and DOPAC (Table 1). $d l$-DPE gave 4 times more intense fluorescence to DOMA than meso-DPE, while it was twice less sensitive to DOPAC; $d l$-DPE was selected because the concentration of DOMA in human urine is much lower than that of DOPAC. Catechol compounds other than DOMA and DOPAC also reacted with $d l$ DPE to produce fluorescence, of which excitation and emission maxima were not very characteristic of the individual compounds (Table 2). Pyrogallol, protocatechuic acid, protocatechualdehyde and esculetin fluoresced intensely under the present reaction conditions using $d l$-DPE, though they did not fluoresce appreciably under the reaction conditions using meso-DPE. ${ }^{23,24}$

Unexpectedly, 4-hydroxy-3-methoxyphenyl compounds also fluoresced under the present reaction conditions, probably because these compounds were oxidatively demethylated to the corresponding o-quinones by oxygen dissolved in the reaction mixture; the compounds did not yield any fluorescence when dissolved oxygen in the reaction mixture was purged by babbling nitrogen gas. Other compounds of biological importance did not fluoresce with $d l$-DPE in the same way as with meso-DPE. ${ }^{24}$

Optimization of reaction conditions. dl-DPE gave 
(A)

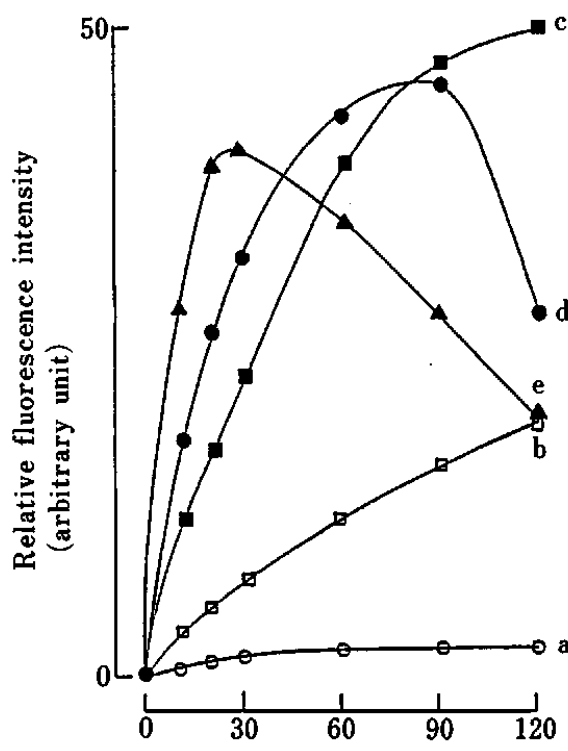

(B)

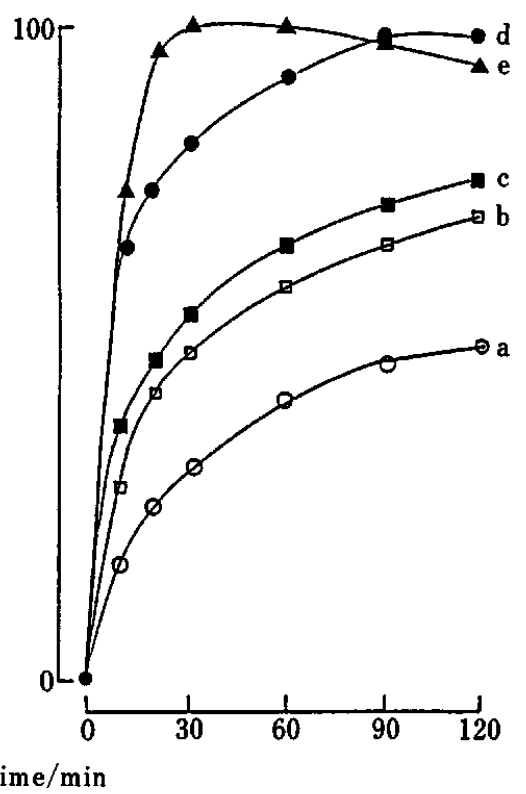

Fig. 2 Effect of the reaction temperature and time on the fluorescence development from (A) DOMA and (B) DOPAC. Concentrations: $1 \mathrm{nmol} \mathrm{ml} \mathrm{l}^{-1}$ each. Temperatures $\left({ }^{\circ} \mathrm{C}\right): \mathrm{a}, 37 ; \mathrm{b}, 60 ; \mathrm{c}, 80 ; \mathrm{d}, 100 ; \mathrm{e}, 120$.

almost maximum fluorescence intensity to DOMA and DOPAC at a concentration of $0.1 \mathrm{M}$. Water-miscible organic solvents, such as methanol, ethanol and 2-propanol accelerated the reaction. Of these solvents, acetonitrile was most effective; $50 \%$ acetonitrile for DOPAC and $30 \%$ acetonitrile for DOMA provided the most intense fluorescence; $30 \%$ was selected for a highly sensitive quantification of DOMA. Almost maximum fluorescence intensities from DOMA and DOPAC were achieved at sodium hydroxide concentrations around 0.5 and $5 \mathrm{mM} ; 5 \mathrm{mM}$ was used for a sensitive measurement of DOMA ( $\mathrm{pH}$ of the reaction mixture, 10.5). The $d l$-DPE derivatives of DOMA and DOPAC fluoresced most intensely also at $\mathrm{pH} \mathrm{10.3-10.5}$.

Though higher temperature allowed fluorescence to develope more rapidly, the prolonged reaction times at high temperature caused a decrease in the fluorescence intensity (Fig. 2), probably due to degradation of the fluorescent products; heating at $100^{\circ} \mathrm{C}$ for $60 \mathrm{~min}$ was optimum. The fluorescence from DOMA and DOPAC under the recommended procedure did not change upon irradiation for $10 \mathrm{~min}$ at their excitation maxima, and was stable for at least $2 \mathrm{~h}$ in daylight.

\section{HPLC of the acidic metabolites}

HPLC separation. Figure $3(\mathrm{~A})$ shows a chromatogram obtained with a standard mixture of the acidic metabolites. An anion-exchanger column (TSK-gel DEAE-2SW) was suitable for the separation of the acidic metabolites. On this column, endogenous catecholamines, their amine metabolites (normetanephrine, metanephrine and 3-methoxytyramine) and alcoholic metabolites (3,4-dihydroxyphenylglycol, 4-
(A)

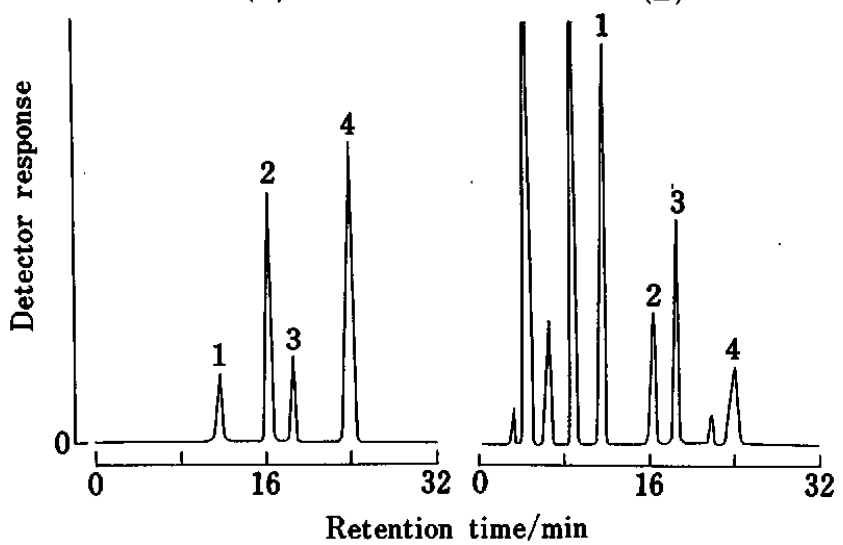

Fig. 3 Chromatograms obtained with (A) a standard mixture of the acidic metabolites and (B) human urine. Peaks: 1, HVA; 2, DOPAC; 3, VMA; 4, DOMA; others, unidentified. Concentrations: (A) $1 \mathrm{nmol} \mathrm{ml}^{-1}$ each; (B) (nmol ml-1) 1, 27.2; $2,2.92 ; 3,19.3 ; 4,1.16$.

hydroxy-3-methoxyphenylglycol and 4-hydroxy-3-methoxyphenylethanol) were eluted much earlier than the acidic metabolites and did not affect the separation of the acidic metabolites.

An acidic solution mixed with acetonitrile was used as a mobile phase. The retention times of the acidic metabolites decreased with decreasing $\mathrm{pH}$ of acidic solutions in the range $2.5-5.0$. Acidic solutions, such as $20 \mathrm{mM}$ citrate buffer (pH $3.2-4.0$ ), $20 \mathrm{mM}$ citrate$10 \mathrm{mM}$ phosphate buffer ( $\mathrm{pH} 2.5-4.0$ ), $10 \mathrm{mM}$ citrate buffer (pH $2.5-4.0$ ) containing $5 \mathrm{mM}$ sodium perchlorate and $25 \mathrm{mM}$ sodium dihydrogenphosphate $(\mathrm{pH}$ 
(A)

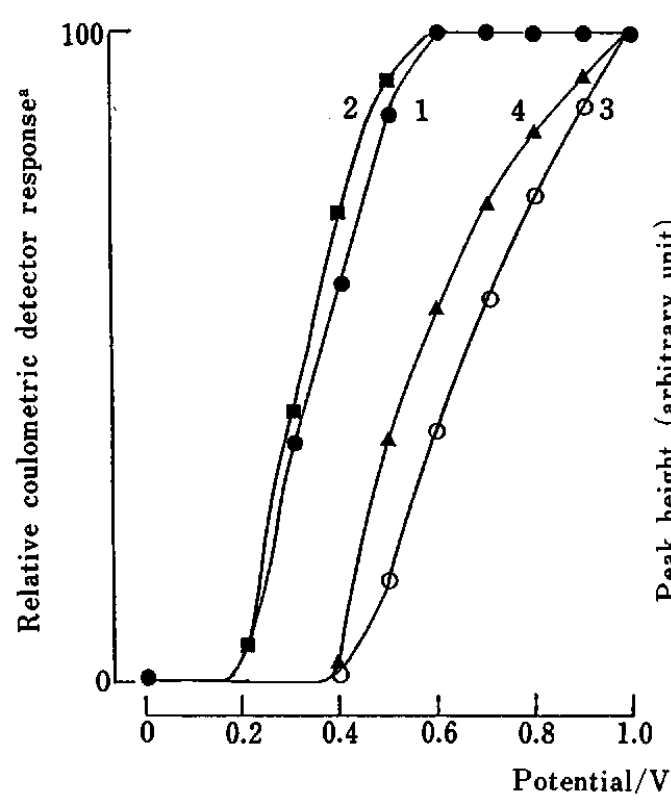

(B)

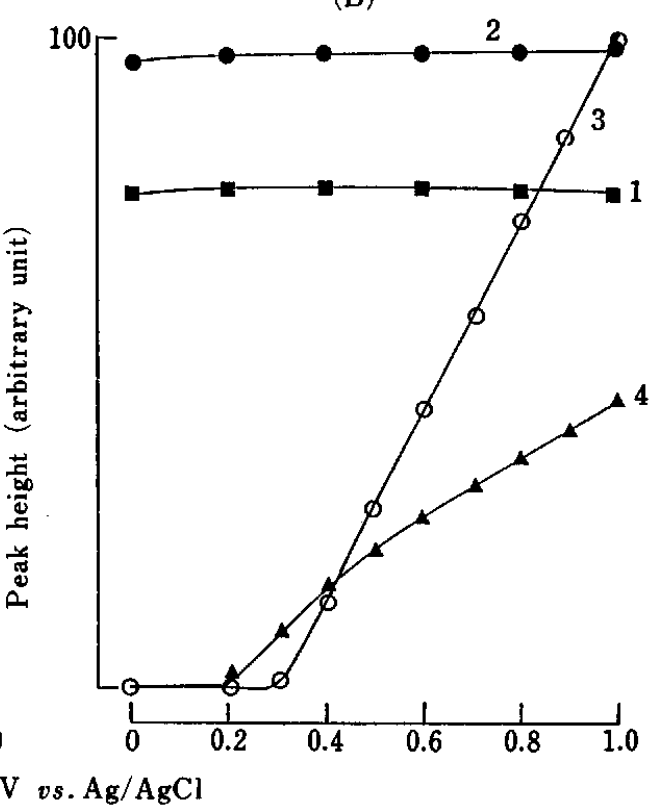

Fig. 4 (A) Hydrodynamic voltammograms ${ }^{\mathrm{a}}$ and (B) effect of applied potential on the fluorescent peak heights for the acidic metabolites. Curves: 1, DOMA; 2, DOPAC; 3, VMA; 4, HVA. Concentrations: $10 \mathrm{nmol} \mathrm{ml}^{-1}$ each. a. Relative coulometric detector response, the oxidative current at $1.0 \mathrm{~V}$ for each compound was taken as 100 .

3.0) have enabled a complete separation of the acidic metabolites. Of these solutions, the dihydrogenphosphate (pH 3.0) afforded the most satisfactory separation from endogenous unidentified compounds when the urine sample was subjected to the HPLC system. The phosphate concentration affected both the retention times and the efficiency of coulometric oxidation. The recommended concentration, $25 \mathrm{mM}$, provided the best results (see Fig. 3(B)).

The addition of acetonitrile to the phosphate solution accelerated the elution. The most satisfactory and rapid separation was attained when a mixture of the phosphate and acetonitrile $(7: 3, v / v)$ was used. EDTA ( $70 \mu \mathrm{M}$ in the phosphate) was added to the mobile phase to protect the coulometric cell and the column from adhesion of metal ions. The retention times of the acidic metabolites were $11.6 \mathrm{~min}$ (HVA), $16.4 \mathrm{~min}$ (DOPAC), $18.6 \mathrm{~min}$ (VMA) and $24.0 \mathrm{~min}$ (DOMA).

Electrochemical oxidation. Figure 4(A) shows hydrodynamic voltammograms of the acidic metabolites. The half-wave potentials of HVA $(0.58 \mathrm{~V})$ and VMA $(0.66 \mathrm{~V})$ were higher than those of DOPAC $(0.35 \mathrm{~V})$ and DOMA $(0.37 \mathrm{~V})$. The fluorescent peak heights for HVA and VMA in the chromatogram were proportional to the respective responses in the coulometric detector with changing the applied potentials within the range $0.2-1.0 \mathrm{~V}$ for HVA and $0.3-1.0 \mathrm{~V}$ for VMA, though those of DOMA and DOPAC were independent of the applied potential (Fig. 4(B)). The concentrations of HVA and VMA in human urine are approximately one order of magnitude higher than those of DOPAC and
DOMA. Therefore, an applied potential of $0.5 \mathrm{~V}$ was selected. Higher applied potentials could afford a more sensitive determination of VMA and HVA.

Fluorescence derivatization reaction. The postcolumn fluorescence derivatization reaction proceeded most rapidly in the presence of 2-propanol. The peak heights for the acidic metabolites increased with increasing 2propanol concentrations in the reagent solutions up to $100 \%$; $d l$-DPE and sodium methylate were dissolved in 2-propanol. The peak heights also increased with increasing $d l$-DPE concentration up to $40 \mathrm{mM} ; 40 \mathrm{mM}$ was used. Sodium methylate was employed for the fluorescence derivatization system, because sodium hydroxide, if used as an alkali, caused the deposition of $d l$-DPE in the reaction coil. The peak heights of DOMA, DOPAC and HVA increased with increasing sodium methylate concentrations ranging from $0.01 \mathrm{M}$ to at least $0.2 \mathrm{M}$. For VMA, however, the maximum peak height was achieved at the concentration of $0.03 \mathrm{M} ; 0.05 \mathrm{M}$ was used for reproducible results (the apparent $\mathrm{pH}$ of the reaction mixture, 10.5). A higher reaction temperature resulted in higher peaks. The selected temperature, $110^{\circ} \mathrm{C}$, gave almost maximum peak heights for the acidic metabolites.

\section{Determination of the acidic metabolites in human urine}

Figure 3(B) shows a typical chromatogram obtained with a urine sample according to the procedure. The peaks for the acidic metabolites were identified as follows; (1) their retention times and fluorescence excitation and emission spectra coincided with those of 
the standard compounds; (2) an increase in peak heights was observed when they were co-chromatographed with the standards; and (3) the fluorescence peak heights for VMA and HVA were proportional to the respective responses in the coulometric device (hydrodynamic voltammograms) with changing applied potentials.

The peaks all disappeared when $d l$-DPE was omitted from the reagent solution for the postcolumn derivatization. Therefore, unidentified peaks in the chromatogram were also attributable to catechol or 4hydroxy-3-methoxyphenyl compounds. Early-eluting of unidentified peaks (the retention times, shorter than $7.5 \mathrm{~min}$ ) could be ascribed to catecholamines and their amine and alcoholic metabolites, while late-eluting unidentified peaks (the retention times, 9.8 and 22.0 min) are probably due to the O-sulfates of the amine metabolites because they had high half-wave potentials (higher than $0.8 \mathrm{~V}$ ). ${ }^{23,26}$

The calibration curves were linear in the ranges $1 \mathrm{pmol}-1.5 \mu \mathrm{mol}$ for DOPAC and HVA, and $1 \mathrm{pmol}-$ $0.5 \mu \mathrm{mol}$ for DOMA and VMA added in 100- $\mu$ l urine sample. The recoveries of the four compounds (10 pmol $-5 \mu \mathrm{mol} \mathrm{ml}^{-1}$ urine each) added to urine samples were actually $100 \%$. The detection limits $\left(\mathrm{fmol} \mathrm{ml}^{-1}\right.$ in urine) at a signal-to-noise ratio of 3 for urine samples were 750 (DOPAC and DOMA) and 1500 (HVA and VMA) (corresponded to 15 and $30 \mathrm{fmol}$ in a $100-\mu l$ injection volume, respectively). The precision of the method was established by repeated determinations $(n=5)$ of a pooled urine sample. The RSD (the compounds and concentrations $\left(\mathrm{pmol} \mathrm{m}^{-1}\right)$ in parentheses) were $2.1 \%$ (DOPAC, 3.88), 2.5\% (HVA, 36.2), $3.3 \%$ (DOMA, 1.55 ) and $3.7 \%$ (VMA, 25.7). The concentrations of the acidic metabolites in urine from healthy persons were assayed by the present method (Table 3). The concentration values agreed well with the previously reported data. ${ }^{17-19}$

Table 3 Amounts of acidic catecholamine-metabolites in 24-h urines from healthy persons

\begin{tabular}{|c|c|c|c|c|c|}
\hline \multirow{2}{*}{ Sex } & \multirow{2}{*}{ Age } & \multicolumn{4}{|c|}{ Amount $/ \mu \mathrm{mol} 24 \mathrm{~h}^{-1}$} \\
\hline & & DOPAC & DOMA & HVA & VMA \\
\hline $\mathbf{M}$ & 38 & 4.87 & 0.95 & 25.2 & 18.7 \\
\hline $\mathbf{M}$ & 32 & 3.88 & 1.55 & 36.2 & 25.7 \\
\hline $\mathbf{M}$ & 27 & 7.02 & 0.50 & 35.1 & 22.4 \\
\hline $\mathbf{M}$ & 26 & 8.19 & 0.95 & 25.8 & 18.7 \\
\hline $\mathbf{M}$ & 24 & 6.56 & 1.18 & 32.3 & 21.4 \\
\hline $\mathbf{M}$ & 24 & 7.19 & 1.59 & 32.8 & 28.4 \\
\hline $\mathbf{F}$ & 29 & 8.24 & 0.81 & 28.5 & 22.7 \\
\hline $\mathbf{F}$ & 24 & 3.76 & 0.97 & 18.8 & 21.9 \\
\hline $\mathbf{F}$ & 23 & 3.37 & 0.51 & 16.8 & 13.2 \\
\hline $\mathbf{F}$ & 21 & 5.12 & 0.34 & 20.5 & 18.5 \\
\hline \multicolumn{2}{|c|}{ Mean } & 5.82 & 0.94 & 27.6 & 21.2 \\
\hline \multicolumn{2}{|c|}{$\pm \mathrm{SD}$} & \pm 2.45 & \pm 0.65 & \pm 10.4 & \pm 8.0 \\
\hline
\end{tabular}

$\mathrm{SD}$, standard deviation.
In conclusion, this method is 20 -times more sensitive to DOMA than the previously reported method using meso-DPE ${ }^{23,24}$, and does not require complicated sample clean-up procedure. Therefore, this method should be useful for biomedical investigations of the acidic metabolites of catecholamines and for clinical studies and diagnosis.

\section{References}

1. P. R. Morrisson, J. T. Edsall and S. G. Miller, J. Am. Chem. Soc., 70, 3103 (1948).

2. M. W. Mosesson and R. A. Umfleet, J. Biol. Chem., 245, 5728 (1970).

3. D. F. Mosher and A. Vaheri, Exp. Cell. Res., 12, 323 (1978).

4. T. M. Saba and E. Jaffe, Am. J. Med., 68, 577 (1980).

5. M. Hayashi and K. M. Yamaji, J. Biol. Chem., 258, 3332 (1983).

6. H. O. Eriksen, I. Clemmensen and M. S. Hansen, Scand. J. Clin. Lab. Invest., 42, 291 (1982).

7. V. De Quattro, D. Wybenga, W. Von Studnitz and S. Brunjes, J. Lab. Clin. Med., 63, 672 (1964).

8. H. Weil-Malherbe, J. Lab. Clin. Med., 69, 1025 (1967).

9. B. D. Drujan, N. Alvarez and J. M. Diaz Borges, Anal.Biochem., 15, 8 (1966).

10. Z. Kahane and P. Vestergaard, Clin. Chim. Acta, 35, 49 (1971).

11. A. Yoshida, M. Yoshioka, T. Tanimura and Z. Tamura, J. Chromatogr., 116, 240 (1976).

12. A. P. J. M. De Jong, R. M. Kok, C. A. Cramers and S. K. Wadman, J. Chromatogr., 382, 19 (1986).

13. C. S. Sastry, V. Gurucharana Das and K. Ekambareswara Rao, Analyst [London], 100, 819 (1985).

14. A. Yoshida, M. Yoshioka, T. Tanimura and Z. Tamura, J. Chromatogr., 227, 162 (1982).

15. K. Yamada, E. Kayama and Y. Aizawa, J. Chromatogr., 223, $176(1981)$

16. S. J. Soldin and J. G. Hill, Clin. Chem., 29, 876 (1983).

17. K. Fujita, K. Maruta, S. Ito and T. Nagatsu, Clin. Chem., 29, 876 (1983).

18. P. Moleman and J. J. M.Borstrok, Clin. Chem., 29, 878 (1983).

19. M. H. Joseph, B. V. Kadam and D. Risby, $J$. Chromatogr., 226, 361 (1981).

20. S. J. Soldin and J. G. Hill, Clin. Chem., 26, 291 (1980).

21. G. M. Anderson, F. C. Feibel and D. J. Cohen, Clin. Chem., 31, 819 (1985).

22. K. Lindgren and N. Rodopoulos, Clin. Chem., 32, 693 (1986).

23. H. Nohta, E. Yamaguchi, Y. Ohkura and H. Watanabe, J. Chromatogr., 467, 237 (1989).

24. H. Nohta, A. Mitsui and Y. Ohkura, Anal. Chim. Acta, 165, 171 (1984).

25. Y. Umegae, H. Nohta and Y. Ohkura, Biomed. Chromatogr., 2, 9 (1987).

26. H. Nohta, E. Yamaguchi, Y. Ohkura and H. Watanabe, J. Chromatogr., 493, 15 (1989). 